\title{
Application of multi-feature based on LMD in fault Feature extraction of
}

\author{
bearing Type \\ Qi Xiaoxuan ${ }^{1, a^{*}} \quad$ Xu Changyuan ${ }^{2, b}$ \\ ${ }^{1}$ School of Electrical Engineering \& Automation, Tianjin University, China \\ ${ }^{2}$ School of information engineering, Shenyang University, China \\ aqi_xx@aliyun.com b960152132@qq.com
}

Key words: kurtosis;approximate entropy;LMD;APEN

Abstract:This paper presents a application of kurtosis and approximate entropy in rolling bearing fault diagnosis.First use the method of LMD adaptive decomposed he rolling bearing vibration signal into different time scales of PF component, then filtered to get PF component, and rebuilt a new weighted optimization reconstruction fusion PF component, finally have the rolling bearing vibration signal feature extraction and realize fault diagnosis.

\section{Introduction}

Approximate entropy, reflects the complexity of a signal.The method can achieve the purpose of the feature extraction,with quantitative mechanical vibration signals by approximate entropy.Mechanical failure is usually a gradual process, the extracted features often be fuzzier, as growth the failure,the complexity of the fault signal is higher and higher.And the complexity of different failure positions is different, so you can use the approximate entropy and classifier to identify faults.But the small fault might be confused with the normal bearing vibration signal, thus, combining kurtosis and approximate entropy theory,so this paper presents a application of kurtosis and approximate entropy in rolling bearing fault diagnosis.First use the method of LMD adaptive decomposed he rolling bearing vibration signal into different time scales of PF component, then filtered to get PF component, and rebuilt a new weighted optimization reconstruction fusion PF component, finally have the rolling bearing vibration signal feature extraction and realize fault diagnosis.

\section{THE DECOMPOSITION OF BEARING FAULT SIGNAL BY LMD}

Due to the machine itself and the outside noise interference,the rolling bearing characteristic frequency is not obvious, Complex composition. LMD is a new iterative approach that decomposes signals into a set of PFs of envelope signals and frequency-modulated signals, and from the PFs the time-varying IF and IA can be derived directly without a Hilbert transform.In LMD, signal $X(t)$ can be expressed as a summation of the PFs and a residue,and PFs is a product of a envelope signal and a purely frequency-modulated signal

$$
X(t)=\sum_{i=1}^{n} P F_{i}(t)+u_{n}(t)
$$

Algorithm 1. The signal adaptive decomposition algorithm of bearing fault Input.Bearing fault vibration signal at work. 
Output.The PF components of different scales.

\section{APPLICATION OF KS-APEN METHOD}

Due to roller bearing fault is smaller, and the degrees to distinguish the signal is smaller when it work normally. At the same time, in order to response more intuitive within each sample value difference degree, the kurtosis and the standard deviation is introduced. .Kurtosis is suitable for surface damage,especially it suit for early fault diagnosis. When the normal work of the bearing vibration signal was close to normal distribution, the kurtosis is between 3-4. With the appearance and development of fault and normal signal probability density increases, the signal amplitude distribution deviates from the normal distribution, normal distribution curve appear skewed or scattered, kurtosis value also increase.As the fault occurs, increasing the complexity of the signal, which makes signal when a failure occurs within each value relative to the size of the work there was a significant difference, the standard deviation of the corresponding signal will also change.In conclusion, through the kurtosis and the standard deviation to determine whether a bearing failure and then by the approximate entropy method for precise positioning failure parts.the Algorithm is as follow,

\section{Algorithm 2 application of KS-APEN method}

Input :Weighted optimization that PFopt.

Output :KS-approximate entropy characteristics.

(1)According to the formula (2) to calculate kurtosis, assume that $X_{i}$ is sampling to a set of data, then the kurtosis $\beta$ is that,

$$
\beta=\frac{1}{N} \sum_{i=1}^{N}\left(X_{i}-\bar{X}\right)^{4}
$$

Setting kurtosis $\beta$ as characteristic vector of the first characteristic

(2)Calculated according to the standard deviation formula (3), assuming $X_{i}$ to a set of data for the sampling, the sample standard deviation for $X_{i}$ is that,

$$
S=\sqrt{\frac{1}{N-1} \sum_{i=1}^{N}\left(X_{i}-\bar{X}\right)^{2}}
$$

Said said the sample average in type $\bar{X}, N$ sample contains the point.Will go through the standard deviation of $S$ as characteristic vector is calculated the second feature of $V$

(3)According to the approximate entropy algorithm mentioned in section 3, calculating the approximate entropy of $A p E n, A p E n$ will get the approximate entropy as $X_{i}(i=1,2, \ldots, N)$ feature vector $V$ third feature.

(4)For each sample in step 1 to step 3 to calculate the corresponding eigenvectors 


$$
V=[\beta, S, A p E n]
$$

The resulting vector feature vector is our requirements, will all the samples according to repeat step 1-4 step operation, can get all the sample feature vector.

\section{APPLICATION EXAMPLE}

The test stand consists of a $2 \mathrm{hp}$ motor(left), a torque encoder (center), a power meter(right). Bearing motor shaft of bearings. The frequency is $12000 \mathrm{~Hz}$, sample length cut at 4096 points, it include 464 samples.

According to the algorithm 1 ,through LMD decomposition of acquisition of bearing vibration signal, we can get 8 PF component and a residual component.Figure 3-5 bearings are given respectively 4 as a result, the working state signal of LMD decomposition is limited by space, only show the first five PF component (black curve).



Figure 1. the LMD decomposition and weight optimization result of normal work

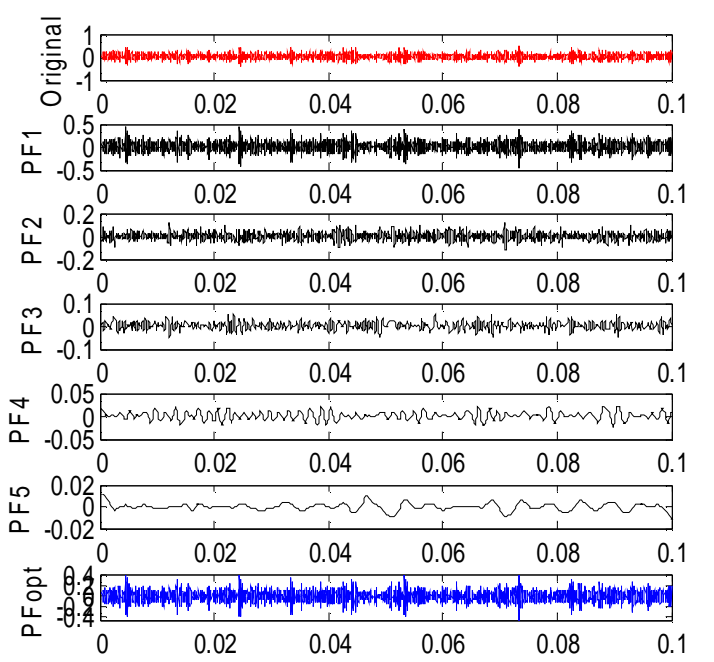

Figure 2. the LMD decomposition and weight optimization results of rolling fault signals

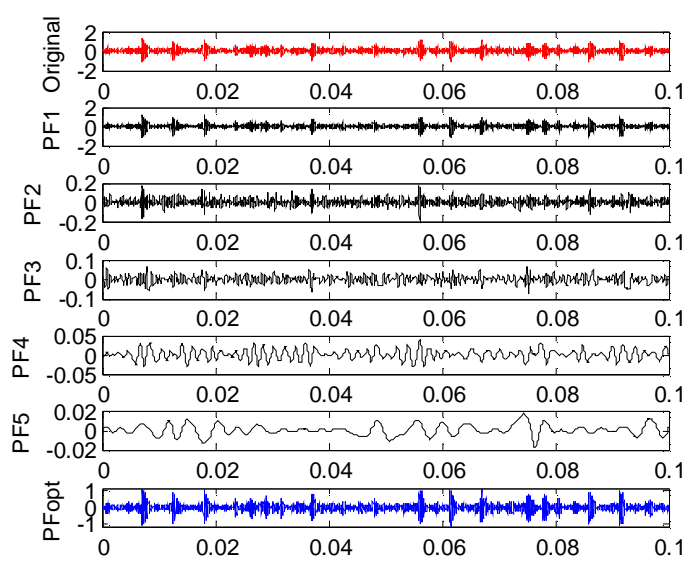

Figure 4. the LMD decomposition and weight optimization results of outer race fault 
Then use the Algorithm 2, four kinds of typical bearing PFopt weighted optimization components were analyzed, and we get the working state of table 1. Analyze the corresponding eigenvectors, of four kinds of typical samples, we can see that kurtosis has a high degree of differentiation on normal work and bearing fault occurs, the failure of rolling element, inner ring and outer ring fault.Standard deviation has high degree of differentiation in the failure of rolling element and the inner ring.Approximate entropy of different failure after failure parts has high degree of differentiation.

Table1 the feature vector of multi-feature

\begin{tabular}{|c|c|c|c|}
\hline \multirow{2}{*}{ work } & \multicolumn{3}{|c|}{ feature vector } \\
\cline { 2 - 4 } & Kurtosis & $\begin{array}{c}\text { the standard } \\
\text { deviation }\end{array}$ & APEN \\
\hline health & 2.5967 & 0.624 & 7.798 \\
\hline rolling fault & 5.2880 & 1.175 & 10.354 \\
\hline inner race fault & 5.6684 & 2.735 & 12.578 \\
\hline outer race fault & 7.8661 & 2.251 & 7.515 \\
\hline
\end{tabular}

\section{Conclusion}

LMD method can make bearing vibration signal which is non-stationary and multi-scale adaptively decompose into a series of single-component signal. It can eliminate the vibration signal which mixed with ambient noise. Analyze the corresponding eigenvectors, of four kinds of typical samples, we can see that kurtosis has a high degree of differentiation on normal work and bearing fault occurs, the failure of rolling element, inner ring and outer ring fault.Standard deviation has high degree of differentiation in the failure of rolling element and the inner ring.Approximate entropy of different failure after failure parts has high degree of differentiation.

\section{References}

[1]Hongchao Wang, Jin Chen, Guangming Dong."Feature extraction of rolling bearing's early weak fault based on EEMD and tunable Q-factor wavelet transform"'[J]. Mechanical Systems and Signal Processing,2014, 48:103-119. IEEE TRANSACTIONS ON BIOMEDICAL ENGINEERING,2005, 52(6):1132-1139.

[2]Jihao Yin, Chao Gao,Xiuping Jia."Wavelet Packet Analysis and Gray Model for Feature Extraction of Hyperspectral Data"[J]. IEEE GEOSCIENCE AND REMOTE SENSING LETTERS,2013,10(4):682-686.

[3]Z.K. Peng, F.L. Chu, "Application of the wavelet transform in machine condition monitoring and fault diagnostics: A review with bibliography"[J].Mechanical Systems and Signal Processing 18 (2) (2004) 199-221.

[4]David B. S.,Havard V.. “Order tracking signal processing for open rotor acoustics”[J].Journal of sound and vibration,2014,333:3818-3830.

[5]Smith J S. "The local mean decomposition method and its application to EEG perception data"[J].Journal of the Royal Society Interface,2005,2(5):443-454.

[6]Bin Yuan,Zengping Chen."Micro-doppler Analysis and Separation Based on Complex Local Mean Decomposition for Aircraft with Fast-rotating Parts in ISAR imaging”[J].IEEE. 2014 